\title{
PERFORMANCE SCHWEIZERISCHER ANLAGESTIFTUNGEN
}

\begin{abstract}
Obwohl Anlagestiftungen einen bedeutenden Anteil der Schweizer Pensionskassenvermögen verwalten, wurde bisher nie eine umfassende Performanceanalyse verfasst. Die vorliegende Arbeit führt erstmals für die Sondervermögen „Aktien Schweiz“ und „Aktien Ausland“ eine solche Untersuchung mit CAPM-basierten Kennzahlen durch, wobei neben den Selektions- auch Timingfähigkeiten betrachtet werden. Zusätzlich wird mit einem Faktormodell analysiert, ob die Sondervermögen „Aktien Schweiz“ einen Value- oder Growth-Stil verfolgen und ob die Wertentwicklung der Sondervermögen „Aktien Ausland“ durch einen Regionenansatz erklärt werden kann. Dabei zeigt sich, dass sich die Anlagestiftungen mit einer Ausnahme relativ nahe am Referenzindex bewegen und nicht durch stark aktives Management auffallen. Bei keiner Anlagestiftung kann eine statistisch signifikante Outperformance nachgewiesen werden, wobei die Performance der Sondervermögen „Aktien Ausland“ schlechter ausfällt als die der „Aktien Schweiz“.
\end{abstract}

\section{$1 \quad$ Einleitung}

Die Performance traditioneller Anlagefonds ist in zahlreichen Studien untersucht worden.[1] Schweizer Anlagestiftungen hingegen waren bisher noch nicht Gegenstand einer umfassenden Performanceanalyse, obwohl die Anlageperformance von Vorsorgegeldern in letzter Zeit zunehmend ins Blickfeld des allgemeinen Interesses gerückt ist.[2] Anlagestiftungen, deren Performance im vorliegenden Artikel untersucht werden soll, stellen einen wichtigen Portfolioanteil der Pensionskassen dar. Es sind steuerbefreite Institutionen, welche fondsähnliche Anlagevermögen, sogenannte Sondervermögen, führen und der Aufsicht des Bundes unterstehen. Das Stiftungsvermögen dient dauernd und ausschliesslich der beruflichen Vorsorge. Mitstifter können alle von der direkten Bundessteuer und der Verrechnungssteuer befreiten Vorsorgeeinrichtungen werden. Das betrifft sowohl Personalvorsorgeeinrichtungen privater und öffentlicher Arbeitgeber als auch Vorsorgeeinrichtungen beruflicher Vereinigungen selbständig- und unselbständig erwerbender Personen.

Besondere Vorteile bieten die Anlagestiftungen kleinen und mittleren Vorsorgeeinrichtungen bezüglich Diversifikation und Kosten, da in der Regel nur grosse Vorsorgeeinrichtungen über die notwendigen Ressourcen verfügen, um ein eigenes Portfoliomanagement zu betreiben. Anlagestiftungen und Anlagefonds werden gemäss der Umfrage von LUSENTI (2002) von rund drei Vierteln der Vorsorgeeinrichtungen eingesetzt.

\footnotetext{
${ }^{1}$ Schweizerisches Institut für Banken und Finanzen, Universität St. Gallen, Rosenbergstrasse 52, 9000 St. Gallen, Tel+41 71 224-7090, Fax+41 71 224-7088. Email: manuel.ammann@unisg.ch, corinne.haeller@student.unisg.ch, heinrich.vonwyss@unisg.ch. Wir danken den untersuchten Anlagestiftungen und Watson Wyatt, Zürich, für die uns zur Verfügung gestellten Daten. Wir danken Michael Genser, Ralf Seiz, Michael Verhofen und dem Gutachter Heinz Zimmermann für hilfreiche Kommentare.
} 
Verschiedene Anlagestiftungen haben sich in der Konferenz der Geschäftsführer von Anlagestiftungen (KGAST) zusammengeschlossen. Die der KGAST angeschlossenen Mitglieder verpflichten sich, spezifischen Richtlinien bezüglich Produktpalette, Produktgestaltung, ethischen Standards, Kontrollstellen und Aufsichtsbehörden zu genügen. Mit der Anlagestiftung Swiss Life ist die Zahl der KGAST-Mitglieder im Jahr 2001 auf zwölf angewachsen. Erweitert hat sich im selben Jahr auch die Produktpalette: Neben den Anlagekategorien „Aktien Schweiz“, „Aktien Ausland“, „Obligationen Schweiz“ und „Obligationen Ausland“, führte die Nachfrage seitens der Anleger zu Angeboten im Bereich Hedge Funds, Private Equity und Multimanagerlösungen. Abgenommen hat im Jahr 2001 hingegen das Gesamtvermögen aller Mitglieder, nämlich von 51.1 Mrd. um netto 7.7\% auf 47.4 Mrd. Dieser Rückgang ist gemäss MÜLLER (2002a) zur Hauptsache auf Wertverminderungen zurückzuführen. Trotz des schwierigen Börsenumfeldes ist es einigen Anlagestiftungen im Jahr 2001 sogar gelungen, bedeutende Nettozugänge zu verzeichnen. Abbildung 1 stellt die Vermögen der einzelnen Stiftungen per 30. Juni 2002 dar. Im ersten Halbjahr 2002 ist das Gesamtvermögen der Mitglieder um weitere 1.3\% gesunken.

\section{Abbildung 1: Gesamtvermögen der Anlagestiftungen per 30.06.2002 (ohne Doppelzählungen)[3]}

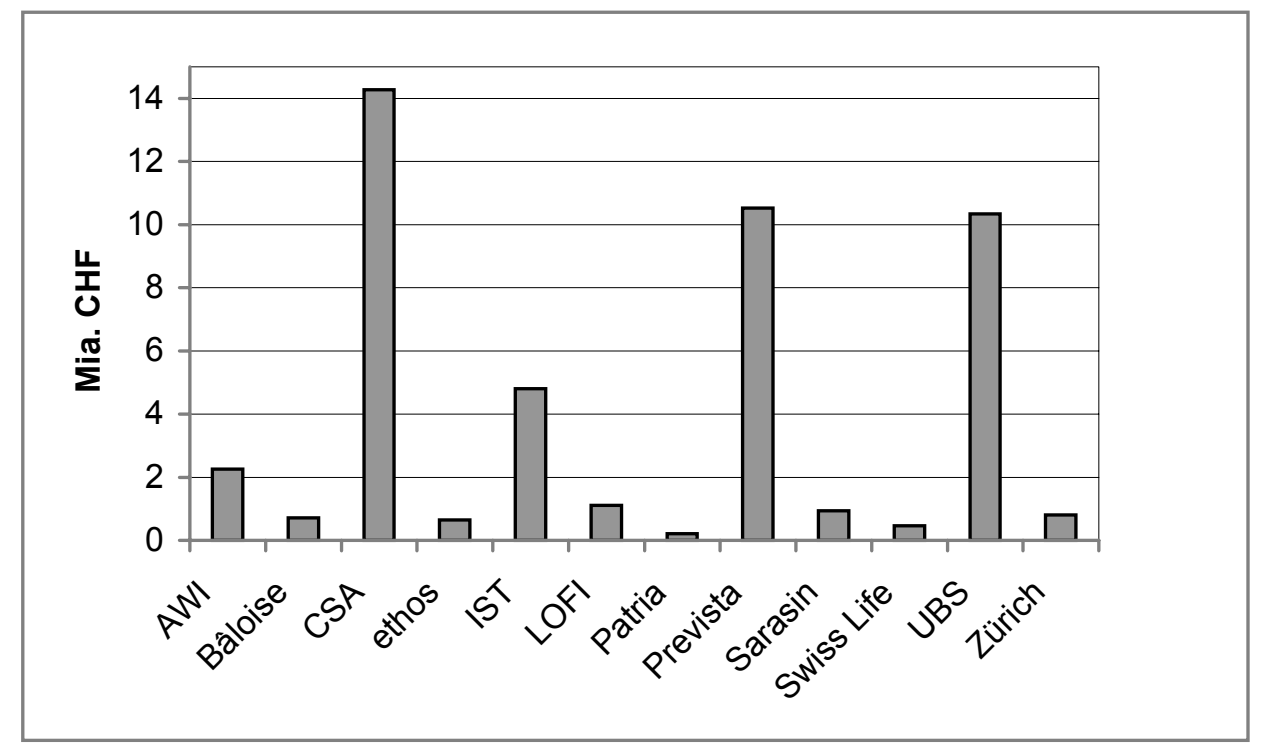

Quelle: Watson Wyatt (2002b)

Die der KGAST angeschlossenen Mitglieder verpflichten sich unter anderem zur einheitlichen Publikation der wichtigsten Kennzahlen ihrer Tätigkeit, insbesondere der Rendite-Performance, des Risikos oder des Vermögens. Die Firma Watson Wyatt berechnet diese Kennzahlen halbjährlich. Ausgewiesen werden die Renditen und Standardabweichungen der Anlagegruppen über die Zeitintervalle von 1, 3, 5 und 10 Jahren.

In diesem Beitrag werden für die fünf grössten Anlagestiftungen sowie die Bâloise die Sondervermögen „Aktien Schweiz“ und „Aktien Ausland“ der KGAST-Anlagestiftungen untersucht. Bei diesen Anlagestiftungen sind sowohl für die „Aktien Schweiz“ als auch für die „Aktien Ausland" genügend lange Zeitreihen verfügbar, so dass auch ein Quervergleich zwischen den Anlagekategorien möglich wird. Diese beiden Anlagekategorien wurden ausgewählt, weil durch ihre homogene Zusammensetzung ein aussagekräftiger Vergleich möglich ist. Auf eine Untersuchung 
der verschiedenen BVG-Mischvermögen musste aufgrund der Datenverfügbarkeit verzichtet werden. Ebenfalls ausgeschlossen wurden in dieser Untersuchung die Vermögen „Obligationen Schweiz“ und „Obligationen Ausland“ sowie die Spezialgruppen (Finanzinstrumente oder spezielle Anlageregionen).

Der folgende Abschnitt beschreibt die Datengrundlage der Sondervermögen und der verwendeten Indizes. Kapitel 3 geht auf Kennzahlen wie Rendite, Volatilität, SHARPE Ratio, JENSEN's Alpha, Beta, TREYNOR Ratio und Information Ratio ein. Ausserdem wird mit dem HENRIKSSON-MERTON-Modell untersucht, ob die Portfoliomanager Timingfähigkeiten aufweisen. Mit dem Asset-Allocation-Modell von SHARPE (1988) und SHARPE (1992) wird in Abschnitt 4 überprüft, ob die Sondervermögen „Aktien Schweiz“ einen Value- oder Growth-Stil verfolgen und ob sich die Renditen der Sondervermögen „Aktien Ausland“ durch einen Regionenansatz erklären lassen. Des Weiteren wird ermittelt, ob einzelne Anlagestiftungen damit signifikante Überschussrenditen erzielen. Schliesslich wird in Kapitel 5 berechnet, ob sich im Beobachtungszeitraum Persistenz in den beiden Anlagekategorien nachweisen lässt. Zum Schluss werden die Resultate zusammengefasst.

\section{Datengrundlage}

Von den untersuchten Anlagestiftungen wurden von Watson Wyatt sowie den einzelnen Anlagestiftungen monatliche Renditedaten der Sondervermögen „Aktien Schweiz“ und „Aktien Ausland“" von Januar 1996 bis September 2002 zur Verfügung gestellt. Bei der Anlagestiftung Winterthur (AWI) ist die Zeitreihe für das Sondervermögen „Aktien Schweiz“ erst ab Mai 1996 erhältlich. Sämtliche Anlagegruppen werden mit Ausnahme des Sondervermögens „Aktien Schweiz“ der AWI aktiv und unter vergleichbaren Anlagerichtlinien verwaltet.

Seit Dezember 1991 werden bei allen Anlagestiftungen einheitliche Bewertungen vorgenommen, was die Vergleichbarkeit der Daten für die vorliegende Beobachtungsperiode gewährleistet. Massgebend für die Performanceberechnung sind die Ausgabekurse an den jeweiligen Stichdaten. Die Renditen werden unter Annahme der Wiederanlage sämtlicher Erträge im Zeitpunkt der Ausschüttung gemessen. Die Verwendung von Ausgabekursen an Stelle von Net Asset Values ist gerechtfertigt, da die Ausgabekurse um einen fixen Prozentsatz von den Net Asset Values abweichen und somit keine Renditeverzerrungen entstehen. Bei Veränderungen dieses fixen Prozentsatzes wird eine entsprechende Adjustierung vorgenommen. Damit widerspiegeln sämtliche ausgewiesenen Zahlen die Nettoperformance nach Kosten. Um sämtliche Kosten bereinigt, würden die Renditen in der Grössenordnung von $0.5 \%$ bis $1 \%$ pro Jahr - gemäss unseren Kostenschätzungen auf der Basis der Jahresberichte der Stiftungen - besser ausfallen.

Die Stichprobe ist frei von Survivorship Bias, da bisher keine KGAST Sondervermögen der Anlagekategorien „Aktien Schweiz“ und „Aktien Ausland“ - beispielsweise aufgrund schlechter Performance - ausgeschieden sind. Damit sind die Renditezeitreihen nicht nach oben verzerrt. Bei den betrachteten Sondervermögen haben auch keine Zusammenführungen von Fonds stattgefunden.

Als Benchmark dient für die „Aktien Schweiz“ Portfolios der Swiss Performance Index (SPI), für die „Aktien Ausland“ der MSCI World ex Switzerland Index. Bei diesen Indizes werden alle Ausschüttungen reinvestiert. Sie erfüllen damit ein notwendiges Kriterium für eine Performance- 
Messung. Im Weiteren stellen die ausgewählten Benchmark-Indizes eine gute Abdeckung der entsprechenden Anlageuniversa sicher und haben sich als eigentliche Standard-Benchmarks etabliert. Auch Watson Wyatt benutzt diese Indizes für den Vergleich der entsprechenden Sondervermögen.

Für die Umsetzung des Asset-Allocation-Modells nennt SHARPE (1992) vier Kriterien für die Wahl des Benchmark-Portfolios: Es soll eine einfache Nachbildung ermöglichen, nicht einfach zu schlagen sein, tiefe Kosten verursachen und schliesslich ex-ante festgelegt werden können. Mit anderen Worten muss es eine echte Investitionsalternative darstellen. Zusätzlich erforderlich ist nach LUCAS und RIEPE (1996), dass die Korrelationen der Benchmark-Portfolios untereinander entweder gering sind oder sich die Standardabweichungen voneinander unterscheiden. So kann von einer ausreichenden Trennschärfe ausgegangen werden. Aus diesen Gründen wird bei den Sondervermögen „Aktien Schweiz“ eine Aufteilung in Value- und Growth-Stil gewählt, weil diese nur in Schweizer Aktienwerten oder im Geldmarkt investiert sind. Gewählt wurden die Datastream-Indizes für Value und Growth (beides Total-Return Indizes) und der 1-Monats CHF Euromarkt-Zinssatz. Diese Indizes entsprechen der Anforderung, dass sich die darin enthaltenen Werte gegenseitig ausschliessen, also jedes Wertpapier lediglich einer Anlagekategorie angehört.

Für die Sondervermögen „Aktien Ausland“ wird untersucht, welchen Einfluss die Regionen Nordamerika, Europa ohne Schweiz und Pazifik auf die Renditen haben. Dafür werden die MSCI-Subindizes MSCI North America, Europe ex Switzerland und Pacific verwendet. Diese werden ebenfalls als Total-Return-Indizes berechnet. Sämtliche MSCI-Indizes wurden in Schweizer Franken umgerechnet.

Als risikofreier Zins für die Berechnungen mit den Schweizer Aktienfonds wurde der 1-Monats Euromarkt-Zinssatz für Schweizer Franken gewählt. Da sämtliche Renditen in CHF berechnet respektive umgerechnet wurden, wird dieser Zinssatz auch für die Berechnungen der Kennzahlen „Aktien Ausland“ benutzt.

\section{Performancemasse}

\subsection{Risiko und Rendite}

Renditen und Volatilitäten für die Sondervermögen „Aktien Schweiz“ sind in Abbildung 2 dargestellt, während sich die dazugehörenden Werte in Tabelle 1 finden. Für sämtliche Berechnungen werden stetige Renditen ( $r$ ) verwendet. Die monatlichen stetigen Renditen werden mit dem Faktor 12 annualisert. Die Volatilität $\left(\sigma_{\mathrm{Pf}}\right)$ wird als Standardabweichung der monatlichen stetigen Renditen gemessen und mit $\sqrt{12}$ annualisiert. 
Abbildung 2: Risiko-Rendite-Profil der Sondervermögen „Aktien Schweiz“

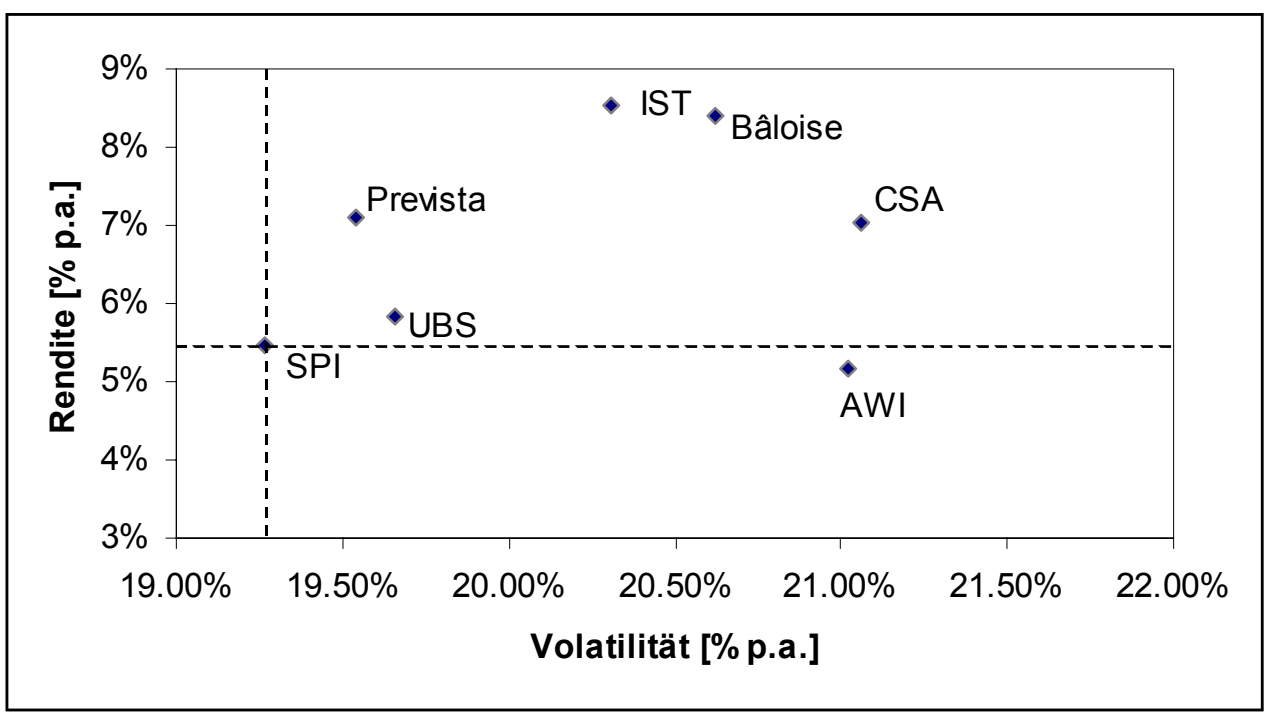

Tabelle 1: Rendite, Volatilität und Korrelation der Anlagestiftungen „Aktien Schweiz““

\begin{tabular}{lrrr}
\hline & Rendite & Volatilität & $\begin{array}{c}\text { Korrelation zum } \\
\text { SPI }\end{array}$ \\
\hline AWI & $5.16 \%$ & $21.02 \%$ & 0.99 \\
Bâloise & $8.38 \%$ & $20.62 \%$ & 0.91 \\
CSA & $7.03 \%$ & $21.06 \%$ & 0.99 \\
IST & $8.52 \%$ & $20.31 \%$ & 0.98 \\
PREVISTA & $7.08 \%$ & $19.54 \%$ & 0.98 \\
UBS & $5.85 \%$ & $19.65 \%$ & 1.00 \\
\hline SPI & $5.46 \%$ & $19.27 \%$ & 1.00 \\
\hline Beobachtungszeitraum: 1. 1996-9.2002, für AWI 5. 1996-9. 2002. \\
Alle Werte sind annualisiert. \\
\hline
\end{tabular}

Die annualisierten Renditen schwanken zwischen $8.52 \%$ für IST und 5.16\% für AWI relativ stark, wobei die Rendite des Benchmark bei 5.46\% liegt. Mit Ausnahme der AWI weisen alle Anlagestiftungen eine höhere Rendite als der SPI Vergleichsindex aus. Die Volatilitäten unterscheiden sich nur wenig voneinander, sie liegen jedoch allesamt höher als diejenige des SPI. Gemessen an den Korrelationen bewegen sich die Anlagestiftungen sehr nahe am Referenzindex. Einzig die Anlagestiftung Bâloise weicht mit einem Wert von $0.91 \mathrm{im}$ Vergleich zu den übrigen etwas stärker ab.

Für die Sondervermögen „Aktien Ausland“ sieht das durch Abbildung 3 (gleicher Massstab wie Abbildung 2) nahegelegte Risiko-Rendite-Profil bedeutend schlechter aus. Alle Anlagestiftungen liegen im Quadranten unten rechts und schneiden damit bezüglich Rendite und Risiko weniger gut ab als der MSCI World Index. Insgesamt liegen die Sondervermögen „Aktien Ausland“ auch deutlich näher zusammen als die Sondervermögen „Aktien Schweiz“. 


\section{Abbildung 3: Risiko-Rendite-Profil der Sondervermögen „Aktien Ausland“}

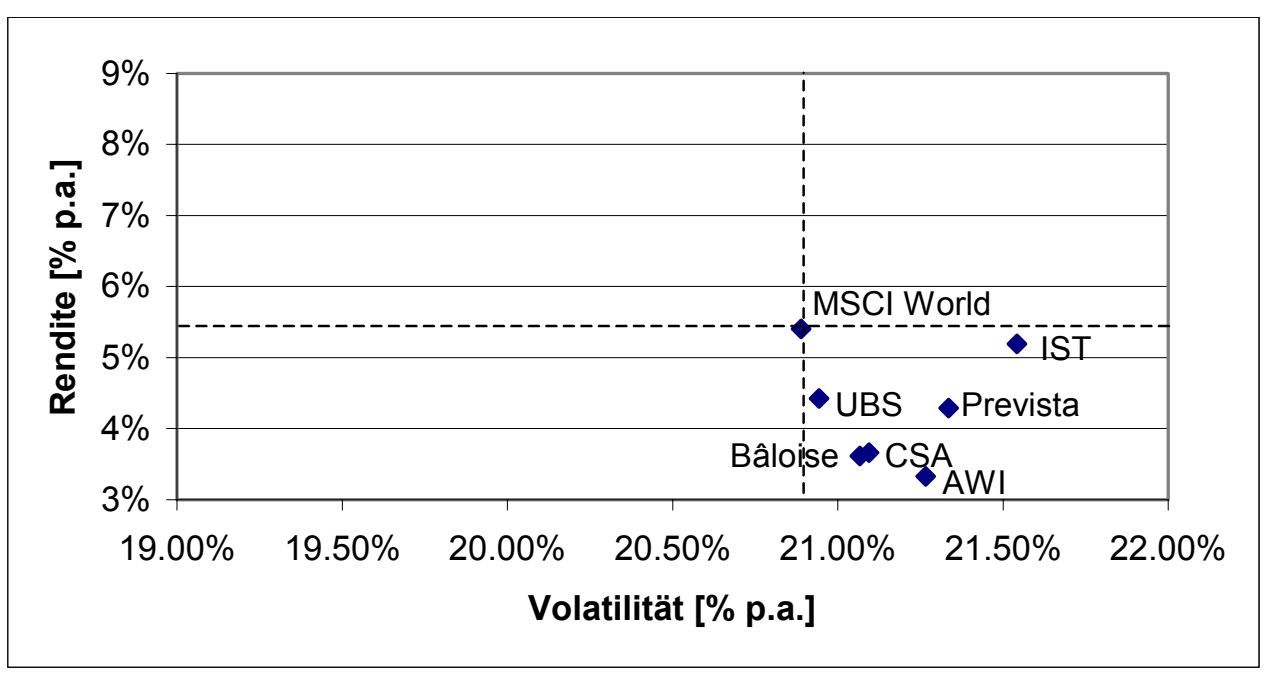

Tabelle 2: Rendite, Volatilität und Korrelation der Anlagestiftungen „Aktien Ausland“

\begin{tabular}{lrrr}
\hline \multicolumn{1}{c}{ Rendite } & Volatilität & $\begin{array}{c}\text { Korrelation zum } \\
\text { MSCI World }\end{array}$ \\
\hline AWI & $3.33 \%$ & $21.27 \%$ & 0.91 \\
Bâloise & $3.62 \%$ & $21.07 \%$ & 0.85 \\
CSA & $3.66 \%$ & $21.10 \%$ & 0.94 \\
IST & $5.19 \%$ & $21.54 \%$ & 0.92 \\
PREVISTA & $4.29 \%$ & $21.34 \%$ & 0.96 \\
UBS & $4.42 \%$ & $20.94 \%$ & 0.94 \\
\hline MSCI World & $5.41 \%$ & $20.89 \%$ & 1.00 \\
\hline \multicolumn{4}{l}{ Beobachtungszeitraum: $1.1996-9.2002}$. \\
\hline
\end{tabular}

Die annualisierten Renditen liegen zwischen 5.19\% für IST und 3.33\% für AWI und damit allesamt unter der Rendite des MSCI World von 5.41\%. Die Volatilitäten sind leicht höher als diejenige des Benchmarks. Die Korrelationen zum MSCI World sind etwas geringer als diejenigen der Sondervermögen „Aktien Schweiz“ zum SPI. Auch bei den Sondervermögen „Aktien Ausland“ ist der Korrelationskoeffizient der Bâloise Anlagestiftung im Vergleich zu den übrigen am kleinsten.

\subsection{Selektivität}

Ausser Rendite, Volatilität und Korrelation werden für die Sondervermögen „Aktien Schweiz“ und „Aktien Ausland“ weitere gebräuchliche Kennzahlen berechnet, nämlich SHARPE Ratio, JENSEN's Alpha, Portfoliobeta, TREYNOR Ratio, Tracking Error und Information Ratio. Das SHARPE Ratio nach SHARPE (1966) berechnet sich folgendermassen:

SHARPE Ratio $_{\mathrm{Pf}}=\frac{\overline{\mathrm{r}_{\mathrm{Pf}}-\overline{\mathrm{r}}_{\mathrm{f}}}}{\sigma_{\mathrm{P}}}$ 
$\overline{\mathrm{r}}_{\mathrm{pf}}-\overline{\mathrm{r}}_{\mathrm{f}}$ ist die durchschnittliche Überschussrendite des betrachteten Portfolios gegenüber dem risikolosen Zinssatz. Das SHARPE Ratio misst eine risikoadjustierte Rendite, wobei als Risiko die Portfoliovolatilität $\sigma_{\mathrm{Pf}}$ dient.

JENSEN's Alpha $\left(\alpha_{\mathrm{Pf}}\right)$ nach JENSEN (1986) und Beta $\left(\beta_{\mathrm{Pf}}\right)$ der Portfolios werden aus folgender Regressionsgleichung gewonnen, wobei $\mathrm{r}_{\mathrm{M}, \mathrm{t}}$ die Benchmarkrendite im Monat $\mathrm{t}$ bezeichnet:

$\mathrm{r}_{\mathrm{Pf}, \mathrm{t}}-\mathrm{r}_{\mathrm{f}, \mathrm{t}}=\alpha_{\mathrm{Pf}}+\beta_{\mathrm{Pf}} \cdot\left(\mathrm{r}_{\mathrm{M}, \mathrm{t}}-\mathrm{r}_{\mathrm{f}, \mathrm{t}}\right)+\varepsilon_{\mathrm{Pf}, \mathrm{t}}$.

Der Tracking Error $\left(\sigma_{\varepsilon, \mathrm{Pf}}\right)$ errechnet sich als Standardabweichung der Störterme $\varepsilon_{\mathrm{Pf}, \mathrm{t}}$.

Das TREYNOR Ratio nach TREYNOR (1965) misst ebenfalls eine risikoadjustierte Überschussrendite. Als Risikomass dient jedoch nicht die Volatilität, sondern das systematische Risiko relativ zum Benchmark (Portfoliobeta, $\beta_{\mathrm{Pf}}$ ). Das TREYNOR Ratio erlaubt somit auch einen Vergleich von unterschiedlich stark diversifizierten Portfolios. Die Definition des TREYNOR Ratios lautet:

TREYNOR Ratio $_{\mathrm{Pf}}=\frac{\overline{\mathrm{r}}_{\mathrm{Pf}}-\overline{\mathrm{r}}_{\mathrm{f}}}{\beta_{\mathrm{Pf}}}$.

Das Information Ratio, auch TREYNOR/BLACK Ratio gemäss TREYNOR und BLACK (1973) oder Apraisal Ratio genannt, dividiert das JENSEN's Alpha durch den Tracking Error und damit durch das fondsspezifische Risiko:

Information Ratio $_{\mathrm{Pf}}=\frac{\alpha_{\mathrm{Pf}}}{\sigma_{\varepsilon}}$.

Das Information Ratio setzt also die erzielte Performance in Relation zur Abweichung vom Benchmark. Es liefert damit eine Antwort auf die Frage, mit welchem Ausmass an aktivem Management und mit wieviel Risiko (relativ zum Benchmark) die Outperformance erzielt wurde. Je höher das Information Ratio, desto effektiver war das aktive Management.

Tabelle 3: Kennzahlen der Anlagestiftungen „Aktien Schweiz“6

\begin{tabular}{lrrrrrrr}
\hline & $\begin{array}{c}\text { SHARPE } \\
\text { Ratio }\end{array}$ & $\begin{array}{c}\text { JENSEN's } \\
\text { Alpha }\end{array}$ & \multicolumn{1}{c}{$\mathbf{t}_{\boldsymbol{\alpha}}$} & \multicolumn{1}{c}{ Beta } & $\begin{array}{c}\text { TREYNOR } \\
\text { Ratio }\end{array}$ & $\begin{array}{c}\text { Tracking } \\
\text { Error }\end{array}$ & $\begin{array}{c}\text { Information } \\
\text { Ratio }\end{array}$ \\
\hline AWI & 0.16 & $-0.53 \%$ & -0.48 & $1.06^{* * *}$ & $3.09 \%$ & $2.74 \%$ & -0.19 \\
Bâloise & 0.32 & $1.80 \%$ & 0.55 & 0.98 & $6.68 \%$ & $8.44 \%$ & 0.21 \\
CSA & 0.25 & $-0.05 \%$ & -0.04 & $1.08^{* * *}$ & $4.79 \%$ & $3.42 \%$ & -0.01 \\
IST & 0.33 & $1.69 \%$ & 0.98 & 1.03 & $6.48 \%$ & $4.43 \%$ & 0.38 \\
PREVISTA & 0.27 & $0.44 \%$ & 0.27 & 0.99 & $5.27 \%$ & $4.17 \%$ & 0.10 \\
UBS & 0.20 & $-0.92 \%$ & -1.29 & 1.02 & $3.92 \%$ & $1.84 \%$ & -0.50 \\
\hline SPI & 0.25 & $0.00 \%$ & - & 1.00 & $4.83 \%$ & $0.00 \%$ & - \\
\hline
\end{tabular}

Beobachtungszeitraum: 1. 1996 - 9. 2002, für AWI 5. 1996 - 9. 2002.

$* * *$ bedeutet Signifikanz auf einem Niveau von $1 \%$.

Alle Werte sind annualisiert.

Tabelle 3 zeigt die verschiedenen Kennzahlen für die Anlagekategorie „Aktien Schweiz“. Da die sich die Volatilitäten nur wenig voneinander unterscheiden, ist die Rangfolge nach SHARPE Ratios identisch mit der Rangfolge nach Renditen. Drei JENSEN's Alphas sind grösser als null, 
wobei diejenigen von Bâloise und IST sogar recht hohe Werte annehmen. Wie die t-Werte jedoch zeigen, ist kein JENSEN's Alpha ist signifikant von null verschieden, so dass keine systematischen Selektionsfähigkeiten der Fondsmanager nachgewiesen werden können. Die Betas von AWI und CSA sind auf einem Signifikanzniveau von 1\% von eins verschieden. Die übrigen liegen alle relativ nahe bei eins, was auf ein im Vergleich zum SPI identisches systematisches Risiko hinweist. Bei den TREYNOR Ratios schneidet die Bâloise Anlagestiftung mit ihrem vergleichsweise tiefen Beta am besten ab, während die AWI auch hier den kleinsten Wert aufweist. Ein vergleichsweise sehr hoher Tracking Error wurde für die Bâloise Anlagestiftung ermittelt, die allerdings damit auch eine relativ hohe Rendite von $8.38 \%$ pro Jahr erzielt hat. Werden die JENSEN's Alphas mit dem Tracking Error für das unsystematische Risiko adjustiert (Information Ratio), so erzielt die IST den höchsten Wert, während die Bâloise mit dem höchsten Alpha wegen des grösseren Tracking Errors auf Platz zwei liegt.

Tabelle 4: Kennzahlen der Anlagestiftungen „Aktien Ausland““

\begin{tabular}{lccccrrr}
\hline & $\begin{array}{c}\text { SHARPE } \\
\text { Ratio }\end{array}$ & $\begin{array}{c}\text { JENSEN's } \\
\text { Alpha }\end{array}$ & \multicolumn{1}{l}{$\mathbf{t}_{\boldsymbol{\alpha}}$} & Beta & $\begin{array}{c}\text { TREYNOR } \\
\text { Ratio }\end{array}$ & $\begin{array}{c}\text { Tracking } \\
\text { Error }\end{array}$ & $\begin{array}{c}\text { Information } \\
\text { Ratio }\end{array}$ \\
\hline AWI & 0.07 & $-1.98 \%$ & -0.80 & 0.97 & $1.51 \%$ & $6.38 \%$ & -0.31 \\
Bâloise & 0.08 & $-1.37 \%$ & -0.35 & $0.88^{* *}$ & $1.99 \%$ & $10.25 \%$ & -0.13 \\
CSA & 0.09 & $-1.73 \%$ & -1.37 & 1.00 & $1.81 \%$ & $3.26 \%$ & -0.53 \\
IST & 0.15 & $-0.28 \%$ & -0.21 & 1.02 & $3.27 \%$ & $3.43 \%$ & -0.08 \\
PREVISTA & 0.11 & $-1.09 \%$ & -0.54 & 0.99 & $2.45 \%$ & $5.22 \%$ & -0.21 \\
UBS & 0.12 & $-0.98 \%$ & -1.38 & 1.00 & $2.57 \%$ & $1.82 \%$ & -0.54 \\
\hline MSCI World & 0.17 & $0.00 \%$ & - & 1.00 & $3.54 \%$ & $0.00 \%$ & - \\
\hline Beobachtungszeitraum: 1. 1996-9. 2002. & & & & & \\
** bedeutet Signifikanz auf einem Niveau von 5\%. \\
Alle Werte sind annualisiert
\end{tabular}

Tabelle 4 stellt die gleichen Kennzahlen für die „Aktien Ausland“ dar. Es fällt auf, dass die SHARPE Ratios deutlich geringer sind als bei den Sondervermögen „Aktien Schweiz“. Sie sind allesamt auch geringer als das SHARPE Ratio des MSCI World Index. Die JENSEN's Alphas sind durchwegs negativ, zum Teil recht deutlich, aber gemäss den t-Werten ohne statistische Signifikanz. Die negativen Alphas führen zu negativen Information Ratios. Mit den Betas um eins erreichen auch die TREYNOR Ratios geringere Werte als der Benchmark. Einzig das Beta der Bâloise Anlagestiftung ist signifikant kleiner als eins. Dies wird auch durch den vergleichsweise hohen Tracking Error bestätigt, der sich hier allerdings nicht in einem positiven JENSEN's Alpha äussert. Insgesamt zeigen die Sondervermögen „Aktien Ausland“ eine Underperformance, die jedoch nicht statistisch signifikant ist.

Wie beispielsweise anhand der JENSEN's Alphas erkennbar ist, fallen die Kennzahlen für die Sondervermögen „Aktien Schweiz“ deutlich besser aus als für die „Aktien Ausland“. Die Anlagestiftungen agieren offenbar erfolgreicher am Schweizer Aktienmarkt als an den ausländischen Märkten. Möglicherweise besitzen die Anlagestiftung für den Schweizer Markt bessere Informationen zur Portfolioselektion als für ausländische Märkte und können deshalb mit Schweizer Aktien eine bessere Performance erzielen.

Der Unterschied in der Performance zwischen den schweizerischen und den ausländischen Portfolios könnte möglicherweise auch durch die höheren Kosten für Management und Administration der ausländischen Vermögenswerte verursacht werden. Das Kostenargument kann auf Grund der Grössenordnung der beobachteten Performanceunterschiede allerdings nur einen Teil der negativen Performance erklären. Schliesslich sei darauf hingewiesen, dass sich die berechneten 
JENSEN's Alphas nicht statistisch signifikant von null unterscheiden. Insofern kann die unterschiedliche Performance auch dem Zufall zugeschrieben werden.

\subsection{Timing}

Mit dem HENRIKSSON-MERTON-Modell von HENRIKSSON und MERTON (1981) wird untersucht, ob die Anlagestiftungen eine Timingstrategie verfolgen. Dieses Modell geht von folgender Regressionsgleichung aus:

$\mathrm{r}_{\mathrm{Pf}, \mathrm{t}}-\mathrm{r}_{\mathrm{f}, \mathrm{t}}=\alpha_{\mathrm{Pf}}+\beta_{\mathrm{Pf}} \cdot\left(\mathrm{r}_{\mathrm{M}, \mathrm{t}}-\mathrm{r}_{\mathrm{f}, \mathrm{t}}\right)+\gamma_{\mathrm{Pf}} \cdot \max \left[0 ; \mathrm{r}_{\mathrm{f}, \mathrm{t}}-\mathrm{r}_{\mathrm{M}, \mathrm{t}}\right]+\varepsilon_{\mathrm{Pf}, \mathrm{t}}$

wobei $\gamma_{P f}$ den Timingkoeffizienten darstellt. Die anderen Variablen haben die gleiche Bedeutung wie oben. Das HENRIKSSON-MERTON-Modell erweitert die CAPM-Regressionsgleichung durch den Payoff einer Putoption auf den Aktienindex, deren Ausübungspreis um die Höhe des risikolosen Zinssatzes über dem gegenwärtigen Indexstand liegt. Falls der risikolose Zins über der Rendite des Aktienmarkts liegt, erhält man die Renditedifferenz, $\mathrm{r}_{\mathrm{f}, \mathrm{t}}-\mathrm{r}_{\mathrm{M}, \mathrm{t}}$, sonst einen Wert von null.

Tabelle 5: HENRIKSSON-MERTON-Modell für die Sondervermögen „Aktien Schweiz“

\begin{tabular}{lccr}
\hline & Alpha & Beta & Gamma \\
\hline AWI & $-1.92 \%$ & $1.10^{\star *}$ & 0.05 \\
Bâloise & $1.15 \%$ & 0.99 & 0.03 \\
CSA & $-0.62 \%$ & $1.09^{\star *}$ & 0.02 \\
IST & $0.42 \%$ & 1.06 & 0.05 \\
PREVISTA & $2.24 \%$ & 0.95 & -0.07 \\
UBS & $-2.39 \% * *$ & $1.05^{\star *}$ & 0.06 \\
\hline SPI & $0.00 \%$ & 1.00 & 0.00 \\
\hline
\end{tabular}

Beobachtungszeitraum: 1. 1996 - 9. 2002, für AWI 5. 1996 - 9. 2002.

** bedeutet Signifikanz auf einem Niveau von $5 \%$.

Alle Werte sind annualisiert

Tabelle 5 zeigt die Resultate der HENRIKSSON-MERTON-Regression für die Sondervermögen „Aktien Schweiz“. Die Alphas fallen mit Ausnahme der Prevista durchwegs geringer aus als in der CAPM-Regression; dasjenige der UBS ist jetzt signifikant negativ. Zusätzlich zu den bereits vorher signifikanten Betas von AWI und CSA ist jetzt auch das Beta der UBS signifikant grösser als eins. Die Timingkoeffizienten (Gamma) sind abgesehen von der Prevista positiv, jedoch nicht signifikant von null verschieden, so dass nicht auf Timingfähigkeiten der Portfoliomanager geschlossen werden kann.

Tabelle 6: HENRIKSSON-MERTON-Modell für die Sondervermögen „Aktien Ausland“

\begin{tabular}{lrcc}
\hline & Alpha & Beta & Gamma \\
\hline AWI & $1.14 \%$ & 0.91 & -0.10 \\
Bâloise & $-6.96 \%$ & 0.99 & 0.19 \\
CSA & $1.43 \%$ & 0.94 & $-0.11^{*}$ \\
IST & $1.42 \%$ & 0.99 & -0.06 \\
PREVISTA & $5.05 \%$ & $0.87^{\star *}$ & $-0.20^{\star *}$ \\
UBS & $0.07 \%$ & 0.98 & -0.04 \\
\hline MSCI World & $0.00 \%$ & 1.00 & 0.00 \\
\hline
\end{tabular}

Beobachtungszeitraum: 1. 1996 - 9. 2002.

*/* bedeutet Signifikanz auf einem Niveau von $10 \% / 5 \%$.

Alle Werte sind annualisiert 
Tabelle 6 präsentiert die Resultate für die Sondervermögen „Aktien Ausland“. Während bei den Sondervermögen „Aktien Schweiz“ die Alphas tendenziell sinken, steigen sie bei den „Aktien Ausland“" an. Nur die Bâloise Anlagestiftung bildet eine Ausnahme: Das Alpha sinkt markant, auch wenn es nicht signifikant von null verschieden ist, während sich das Beta an eins annähert. Dafür ist jetzt das Beta der Prevista signifikant kleiner als eins, während das Alpha deutlich gestiegen ist. Die Timingkoeffizienten von CSA und Prevista sind auf dem 10\% respektive 5\% Niveau signifikant und negativ. Dies bedeutet, dass bei diesen beiden Sondervermögen systematisch „falsches“ Timing betrieben wurde.

Der negative Timingkoeffizient kann verschiedene Ursachen haben: Er kann einerseits auf unterschiedliche Geldbestände im Fondsvermögen zurückzuführen sein. Müssen in einem sinkenden Aktienmarkt viele Anteile zurückgenommen werden, so könnte der Cashbestand fallen, was sich in einer höheren Sensitivität gegenüber dem Index äusser würde. Sind umgekehrt in steigenden Aktienmärkten wegen Kapitalzuflüssen die Cashbestände grösser, resultiert ein negativer Timingerfolg. Andererseits kann die negative Timing-Performance auch durch Umschichtungen im Aktienportfolio selbst verursacht werden, indem während steigenden Aktienmärkten das Portfolio ein geringeres Beta aufweist als in sinkenden Märkten.

\section{Faktormodell}

Ausgangspunkt zur Entwicklung von Asset-Allocation-Modellen stellt die Beobachtung dar, dass der Grossteil der Portfoliorenditen (und deren Schwankungen) durch die strategische AssetAllocation bestimmt wird, das heisst durch die Aufteilung des Kapitals auf wesentliche Vermögenskategorien. BRINSON, et al. (1986) und BRINSON et al. (1991) haben dies in ihren Untersuchungen über amerikanische Pensionsfonds nachgewiesen. Die Untersuchung von ZIMMERMANN et al. (1996) für eine Schweizer Pensionskasse bestätigt diese Befunde, wenn auch mit weniger ausgeprägten Ergebnissen. Für Schweizer Pensionskassen stellen die Anlagevehikel der Anlagestiftungen lediglich einen Bestandteil eines umfassenden Portfolios dar, weshalb für sie die Identifikation des Anlagestils und eine Risikoanalyse besonders wichtig sind: Sie kennen zwar die deklarierte Anlagepolitik und damit den globalen Risikorahmen, aber nicht die effektive Umsetzung. Diese hat einen entscheidenden Einfluss auf die Performance und die Risikostruktur.

Wenn also die Renditen hauptsächlich durch die langfristig im Rahmen der Anlagepolitik vorgegebenen Aufteilung des Sondervermögens beziehungsweise durch den Anlagestil bestimmt werden, ist dieser Renditeanteil auch für eine Pensionskasse, die nicht über besondere Selektionsoder Timing-Fähigkeiten verfügt, erzielbar. Die zusätzliche Rendite aus aktivem Management beruht auf Abweichungen von der Vermögensgewichtung - auch als Fondsstil bezeichnet - und auf Selektionsfähigkeiten.

\subsection{SHARPE's Investment Style Modell}

Im vorliegenden Artikel wird auf das Modell von SHARPE (1988) und SHARPE (1992) zurückgegriffen.[4] Kern dieses Ansatzes bildet die Identifikation der Asset-Allocation eines Fonds, ohne dass Daten über die Portfoliozusammensetzung bekannt sein müssen. Zur Bestimmung des Stils wird ermittelt, wie die Fondsrenditen von den Renditen der Faktoren respektive der Assetkategorien determiniert werden. Misst man die aktive Rendite eines Fonds nämlich als Zusatzrendi- 
te gegenüber dem Marktportfolio, wird die Performance unter Umständen durch den gewählten Investment Style des Fonds überschattet. Die Zusatzrendite wird nämlich unter Umständen durch einen vom Marktindex abweichenden passiven Asset-Mix erzielt und nicht durch ein aktives Management. Das Faktormodell wird mit folgender Regressionsgleichung geschätzt:

$\mathrm{r}_{\mathrm{Pf}, \mathrm{t}}=\mathrm{b}_{\mathrm{Pf}, \mathrm{l}} \cdot \mathrm{F}_{1, \mathrm{t}}+\mathrm{b}_{\mathrm{Pf}, 2} \cdot \mathrm{F}_{2, \mathrm{t}}+\ldots+\mathrm{b}_{\mathrm{Pf}, \mathrm{n}} \cdot \mathrm{F}_{\mathrm{n}, \mathrm{t}}+\varepsilon_{\mathrm{t}} \cdot$

Die Sensitivitäten $b_{P f, 1}$ bis $b_{P f, n}$ eines Multifaktormodells werden dabei durch eine Regression der erzielten Rendite $\mathrm{r}_{\mathrm{Pf}}$ auf die Faktoren 1 bis $\mathrm{n}\left(\mathrm{F}_{1}\right.$ bis $\left.\mathrm{F}_{\mathrm{n}}\right)$ ermittelt. Der Nachteil bei dieser Berechnungsart ist, dass die damit geschätzten Koeffizienten nicht direkt als Portfoliogewichte der entsprechenden Assetkategorie beziehungsweise eines bestimmten Faktors interpretiert werden können, weil sich erstens die Koeffizienten nicht zu eins aufaddieren und weil sie zweitens auch negative Werte annehmen können.[5] Art. 56a Abs. IV BVV 2 schreibt den Vorsorgeeinrichtungen vor, dass durch derivative Instrumente implizit weder Leerverkäufe noch ein Hebel auf das Gesamtvermögen der Pensionskasse entstehen dürfen. Weil damit bei den Anlagestiftungen keine Nettoshortpositionen zugelassen sind, wird die Ermittlung des Fondsstils analog zur Vorgehensweise von SHARPE (1992) unter Berücksichtigung zweier Nebenbedingungen, vorgenommen:

$$
\min _{\mathrm{b}} \sum_{\mathrm{t}=1}^{\mathrm{T}}\left(\mathrm{r}_{\mathrm{Pf}, \mathrm{t}}-\mathrm{F}_{\mathrm{t}}^{\prime} \cdot \mathrm{b}_{\mathrm{Pf}}\right)^{2}
$$

mit den Nebenbedingungen

$\sum_{\mathrm{i}=1}^{\mathrm{n}} \mathrm{b}_{\mathrm{Pf}, \mathrm{i}}=1$ und $\mathrm{b}_{\mathrm{Pf}, \mathrm{i}} \geq 0$.

Umgesetzt wurde dieses Optimierungsproblem mit Hilfe der Reparametrisierung von MÜLLER (2002b). Ein rollierendes Fenster von 36 Monaten ermöglicht eine erste Schätzung der Faktoren für Januar 1999, die dann für jeden folgenden Monat aktualisiert wird.

Die aus dem Fondsstil resultierende Rendite ergibt sich somit durch die Rendite eines Portfolios, das mit den Anteilen $b_{\mathrm{Pff}, \mathrm{i}}$ in die n Asset-Klassen investiert ist. Dieser Anteil der Rendite resultiert aus dem Fondsstil. Wichtig ist es, sich bei diesem Modell bewusst zu sein, dass es nicht den Anspruch erhebt, die tatsächliche Komposition der bewerteten Anlagen wiederzugeben. Laut SHARPE (1988) besagt es nur, dass sich die Rendite des bewerteten Portfolios so verhält, als $o b$ das Portfolio sich gemäss dem ermittelten Stil zusammensetzt. Die Koeffizienten drücken eine konstante Kombination der Vermögenskategorien aus, die der Renditeentwicklung des Fonds unter Berücksichtigung der Restriktionen im entsprechenden Zeitraum am nächsten gekommen ist. Natürlich ist zu erwarten, dass die Gewichte zumindest eine tendenzielle Annäherung an die reale Aufteilung der Assets in den Sondervermögen ermöglichen. 


\subsection{Stilanalyse der Sondervermögen „Aktien Schweiz“}

\section{Abbildung 4: Faktoranalyse für die Sondervermögen „Aktien Schweiz“}
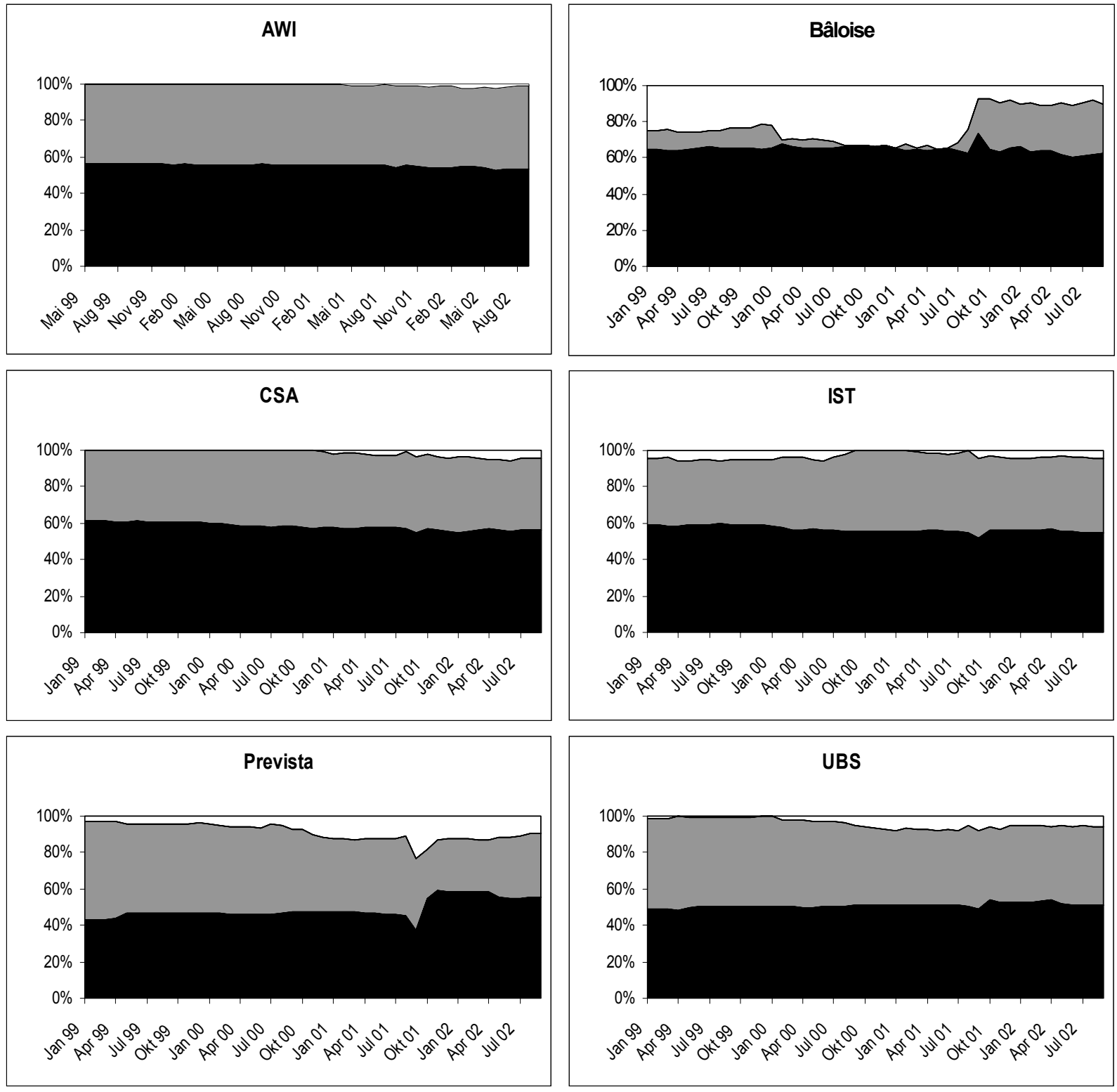

\section{Value $\square$ Growth $\square$ Zins}

Mit der Faktoranalyse wird für die Sondervermögen „Aktien Schweiz“ untersucht, ob die Anlagestiftungen einen Value- oder Growth-Stil verfolgen. Die Resultate werden in Abbildung 4 ausgewiesen. Die einzelnen Sondervermögen zeigen relativ konstante Verhältnisse des Value Anteils von $40 \%$ bis $60 \%$. Bei der Bâloise Anlagestiftung treten jedoch, wie sich bereits aus dem Tracking Error vermuten lässt, grössere Abweichungen auf: Der Growth-Anteil ist relativ gering, während der Geldmarkt bis September 2001 um 30\% ausmacht. Ab September 2001 ist die Aufteilung in Value- und Growth-Stil mit den anderen Anlagestiftungen vergleichbar. Der durchschnitt- 
liche $\mathrm{R}^{2}$-Wert[6] ist hier mit 0.80 im Vergleich zu den übrigen Anlagestiftungen mit Werten zwischen 0.91 und 0.99 auch etwas geringer. Um den September 2001 treten Verwerfungen im Verhältnis von Value- und Growth-Stil sowie Zins bei Bâloise und Prevista auf. In geringerem Ausmass ist dieser Effekt auch bei IST und UBS erkennbar.

Grosse Bewegungen im Verteilungsflächengraphen können gemäss LUCAS und RIEPE (1996) mehrere Ursachen haben:

- Stark aktives Management:

Markttiming oder Sektorrotationen können durch schwankende Gewichte im Flächendiagramm zum Ausdruck kommen. Als Folge ändert der Benchmark, der die Returns am besten erklärt. Diese starken Stiländerungen offenbaren sich bei der Bâloise Anlagestiftung im hohen Tracking Error.

- Veränderungen in den Einzeltiteln oder Anlageklassen selbst:

Sind die Turnovers tief und schwanken die Gewichte trotzdem stark, kann die Ursache in den Einzeltiteln oder Anlageklassen selbst liegen. Fonds mit hohen Konzentrationen in einzelnen Anlagen sind Kandidaten für diese Schwankungsart im Flächengraphen. Die Anlagevorschriften des BVV 2 verhindern gerade grosse Konzentrationen in einzelne Anlagen oder Sektoren, weshalb dieser Grund hier nicht an erster Stelle möglicher Ursachen

- \$olah korrelierte passive Indizes:

$\mathrm{Zu}$ hohe Korrelationen der Benchmarks führen zu einer Oszillation der Gewichte. Analog führt eine unvollständige Kombination von Benchmarks zu einer Regression, die den Benchmark über die Zeit nicht konsistent erklären kann. Die Nebenbedingung, dass sich die Gewichte zu eins aufaddieren müssen, führt dann zu einer Regression, die zwischen denjenigen Indizes hin und her wechselt, die temporär die beste Schätzung bringen. Dies widerspiegelt sich in einem tiefen $\mathrm{R}^{2}$. In der vorliegenden Untersuchung sind die Indizes so gewählt, dass dieses Problem nicht entstehen kann.

Zur Messung der auf aktives Management zurückzuführenden Rendite wird mit Hilfe der im Zeitraum $t-36$ bis $t-1$ durch die Stilanalyse ermittelten $b_{P f, 1}$ bis $b_{P f, n}$ die gewichtete Durchschnittsrendite des Stilportfolios im Zeitpunkt $t$ berechnet. Die Durchschnittsrendite liefert somit den Benchmark. Die Differenz der Fondsrendite im Monat $t$ und dieser dem Fondsstil entsprechenden Benchmarkrendite ergibt die Selektionsrendite im Monat $t$ :

Selektionsrendite $_{\mathrm{Pf}, \mathrm{t}}=\mathrm{r}_{\mathrm{Pf}, \mathrm{t}}-\sum_{\mathrm{i}=1}^{\mathrm{n}} \mathrm{b}_{\mathrm{Pf}, \mathrm{i}, \mathrm{t}} \cdot \mathrm{F}_{\mathrm{i}, \mathrm{t}}$

Die Selektionsrendite beim Asset-Allocation-Modell umfasst auch Abweichungen vom Managementstil, die zum Beispiel aus Timing oder Rotation zwischen den Vermögenskategorien resultieren. In diesem Fall ist also eher die Gesamtperformance des Managers angesprochen. Die Abweichungen vom Benchmark sind damit laut SHARPE (1992) auch als Tracking Error interpretierbar.

Positiv an diesem Ansatz ist neben der individuellen Berücksichtigung des Fondsstils und dem Einbezug der Leerverkaufsrestriktion weiter zu werten, dass bei der Benchmarkkonstruktion grundsätzlich von investierbaren Portfolios ausgegangen werden kann. Ausserdem wird der passive Benchmark für den Monat $t$ ex-ante und out-of-sample aus den drei vorhergehenden Jahren geschätzt. 
Negativ fällt ins Gewicht, dass der Benchmark nicht stabil ist, weil er für jede Periode rollierend neu festgelegt wird. Man kann den Benchmark zwar stabil werden lassen, indem man für die ganze Betrachtungsperiode ein Stilportfolio bestimmt. Damit gibt man aber den Vorteil des exante, out-of-sample definierten Benchmarks auf. Dafür können bei der in-sample Technik die Resultate mit anderen externen Verfahren verglichen werden, die auch in-sample berechnet wurden.

Tabelle 7: Selektionsrenditen für „Aktien Schweiz“6

\begin{tabular}{lr}
\hline \multicolumn{2}{c}{ Selektionsrenditen } \\
\hline AWI & $-1.30 \%$ \\
Bâloise & $1.76 \%$ \\
CSA & $-0.81 \%$ \\
IST & $0.74 \%$ \\
Prevista & $0.26 \%$ \\
UBS & $-1.36 \%$ \\
\hline Beobachtungszeitraum: 1. 1996 - 9. 2002, für AWI 5. 1996- -9.2002. \\
Alle Werte sind annualisiert.
\end{tabular}

Die Selektionsrenditen in Tabelle 7 zeigen Werte zwischen $-1.36 \%$ und $1.76 \%$ pro Jahr. Die grösste Selektionsrendite erzielt die Bâloise, was auf deren stark aktives Management zurückzuführen ist. Allerdings ist kein Wert signifikant von null verschieden. Dies weist einerseits darauf hin, dass keine grossen Abweichungen vom Anlagestil des Benchmarks auftraten sowie andererseits, dass die allfälligen Abweichungen nicht mit einer höheren Rendite einher gingen. Im Vergleich zu den Kennzahlen aus Abschnitt drei ergeben die Selektionsrenditen die gleiche Rangfolge der Anlagestiftungen wie die JENSEN's Alphas; diese sind jedoch höher. Die tieferen Selektionsrenditen im Vergleich zu den JENSEN's Alphas deuten darauf hin, dass die Abweichung vom Benchmark-Stil nicht zu einer Outperformance beitrug. Die gleiche Rangfolge zeigt, dass es keiner Anlagestiftung gelungen ist, durch gezielte Wahl eines eigenen Stils die relative Performance im Vergleich zu den anderen Stiftungen zu ihren Gunsten zu beeinflussen. 


\subsection{Stilanalyse der Sondervermögen „Aktien Ausland“}

Abbildung 5: Faktoranalyse für die Sondervermögen „Aktien Ausland“
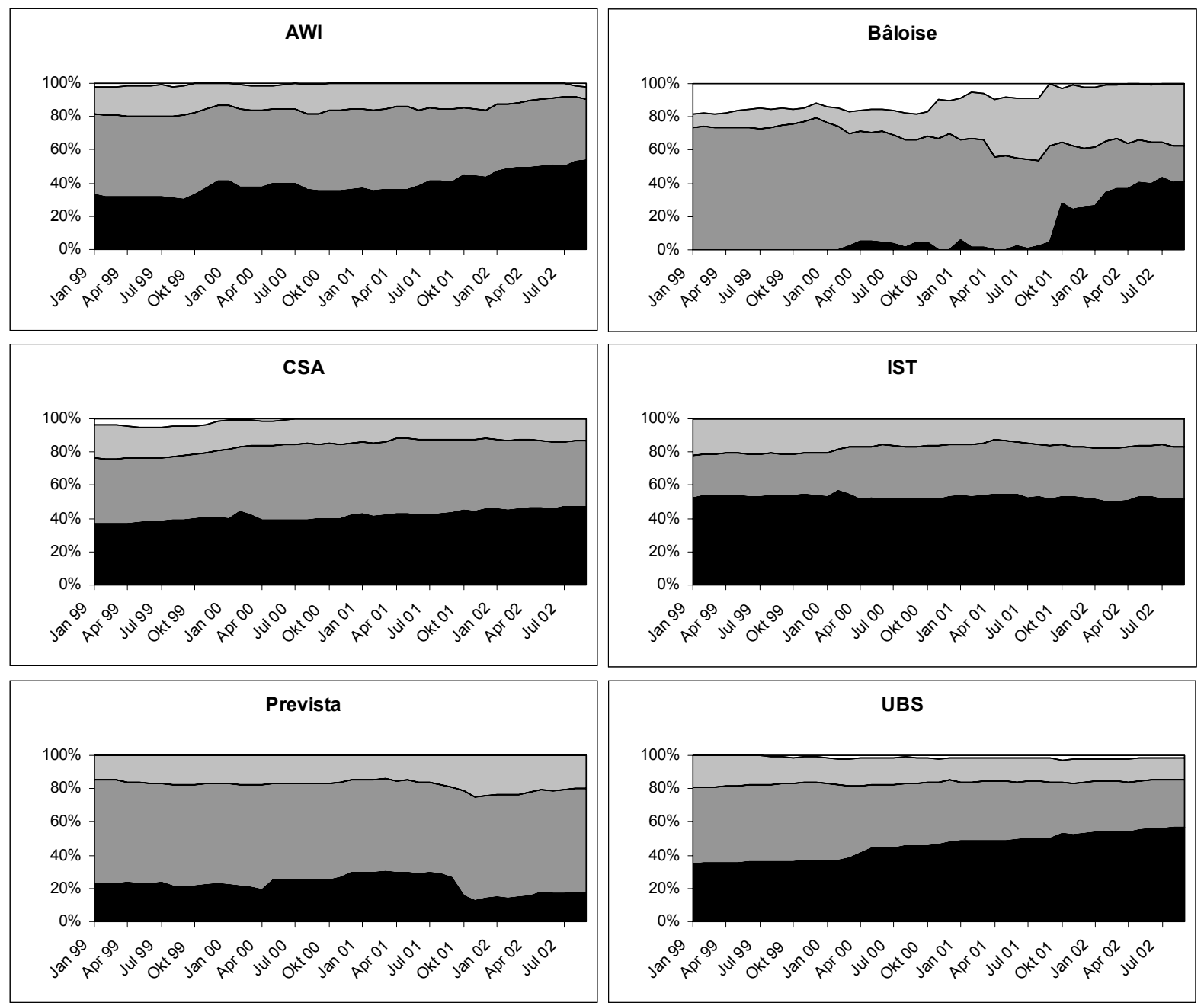

\section{Nordamerika $\square$ Europa ohne Schweiz $\square$ Asien/Pazifik $\square$ Zins}

Für die „Aktien Ausland“ wurde ein Regionenansatz gewählt: Die Renditen wurden in Nordamerika, Europa ohne Schweiz, Asien und Pazifik sowie den risikolosen Zinssatz aufgeteilt. Abbildung 5 zeigt die Resultate der Stilanalyse. Für die Anlagestiftung Bâloise ist zeitweise ein relativ hoher Anteile des Geldmarktes von etwa $20 \%$ zu beobachten, während der amerikanische Markt zu Beginn der Zeitreihe stark untergewichtet wurde. Die Bâloise Anlagestiftung weist auch für die „Aktien Ausland“ den geringsten durchschnittlichen $\mathrm{R}^{2}$-Wert von 0.73 auf. Die übrigen durchschnittlichen $\mathrm{R}^{2}$-Werte liegen zwischen 0.88 und 0.99. Der Effekt des 11. Septembers 2001 kann sowohl bei der Bâloise als auch bei der Prevista beobachtet werden, auch wenn sich die Anteile im amerikanischen Aktienmarkt innerhalb der beiden Stiftungen gegenläufig entwickeln. 
Tabelle 8: Selektionsrenditen für „Aktien Ausland““

\begin{tabular}{lc}
\hline \multicolumn{2}{c}{ Selektionsrenditen } \\
\hline AWI & $1.01 \%$ \\
Bâloise & $0.15 \%$ \\
CSA & $-1.78 \%$ \\
IST & $-1.69 \%$ \\
Prevista & $-0.69 \%$ \\
UBS & $-1.46 \%{ }^{*}$ \\
\hline Beobachtungszeitraum: 1. 1996 - 9. 2002. \\
* bedeutet Signifikanz auf einem Niveau von 10\%. \\
Alle Werte sind annualisiert. \\
\hline
\end{tabular}

Die Selektionsrenditen sind in Tabelle 8 dargestellt. Sie sind mehrheitlich negativ. Die mit Abstand beste Selektionsrendite hat die AWI Anlagestiftung erzielt; sie hat mit $6.38 \%$ auch einen relativ hohen Tracking Error. Der grösste Tracking Error der Bâloise schlägt sich bei den „Aktien Ausland“ im Gegensatz zu den „Aktien Schweiz“ nicht in einer besonders grossen Selektionsrendite nieder, und obwohl die UBS gemäss Tracking Error und Beta dem MSCI World am stärksten gefolgt ist, erzielt sie eine auf dem 10\% Niveau signifikant negative Selektionsrendite.

Insgesamt lässt sich weder für die „Aktien Schweiz“ noch für die „Aktien Ausland“ (mit Ausnahme der UBS) eine statistisch signifikante Über- oder Unter-Performance gegenüber dem Benchmarks durch besondere Selektionsfähigkeiten bezüglich des Anlagestils feststellen.

\section{$5 \quad$ Persistenz der Performance}

Mit einer Persistenzanalyse wird untersucht, ob die Performance über die Zeit konstant ist und somit auf deren zukünftige Entwicklung geschlossen werden kann. Das würde bedeuten, dass zum Beispiel die Outperformer der letzten Periode zu den Outperformern der Folgeperiode gehören und sich dies gezielt in der Anlagestrategie ausnützen liesse.

Bisherige empirische Studien ergeben kein einheitliches Bild über das Vorliegen von Persistenz bei Anlagefonds. GRINBLATT und TITMAN (1992) finden beispielsweise eine positive Persistenz. Die Ergebnisse sind konsistent mit persistenten Gebühren- und Transaktionskostendifferenzen, obschon die Autoren diese nicht als alleinige Erklärung für die Resultate ihrer Studie verantwortlich machen. Gewisse persistente Strukturen im Anlageerfolg finden auch GOETZMANN und IBBOTSON (1994) und HENDRICKS et al. (1993). Diese Persistenz wurde aber entweder dem Survivorship Bias oder unzulänglichen Benchmarks beigemessen. Die Studie von CARHART (1997) deutet darauf hin, dass Persistenz der Fonds-Performance nicht auf Stock-PickingFähigkeiten der Manager zurückgeführt werden kann. Zu ähnlichen Ergebnissen kamen CHRISTOPHERSON et al. (1998) bei der Performanceanalyse von Rentenfonds.

Für die vorliegende Stichprobe wurde eine Unterteilung in zwei ungefähr gleich lange Subperioden vorgenommen. Die 1. Periode beinhaltet die Rendite- und Benchmarkzeitreihen von Januar 1996 bis April 1999 und ist für die „Aktien Schweiz“ in Tabelle 9 bei der jeweiligen Anlagestiftung in der ersten Zeile dargestellt. In der zweiten Zeile ist die 2. Periode von Mai 1999 bis September $2002 \mathrm{zu}$ finden. Falls persistente Überperformance vorliegen würde, wären die JENSEN's Alphas über beide Perioden signifikant. 
Tabelle 9: Persistenz für „Aktien Schweiz“

\begin{tabular}{|c|c|c|c|c|c|c|c|c|}
\hline & Rendite & Volatilität & $\begin{array}{c}\text { SHARPE } \\
\text { Ratio }\end{array}$ & $\begin{array}{c}\text { JENSEN's } \\
\text { Alpha }\end{array}$ & Beta & $\begin{array}{c}\text { TREYNOR } \\
\text { Ratio } \\
\end{array}$ & $\begin{array}{c}\text { Tracking } \\
\text { Error }\end{array}$ & $\begin{array}{c}\text { Information } \\
\text { Ratio }\end{array}$ \\
\hline \multirow{2}{*}{ AWI } & $24.08 \%$ & $24.30 \%$ & 0.93 & $-0.99 \%$ & $1.09^{\star \star \star *}$ & $20.76 \%$ & $2.85 \%$ & -0.35 \\
\hline & $-11.45 \%$ & $16.50 \%$ & -0.82 & $-1.04 \%$ & 1.02 & $-13.30 \%$ & $2.55 \%$ & -0.41 \\
\hline \multirow{2}{*}{ Bâloise } & $24.69 \%$ & $21.70 \%$ & 1.07 & $2.79 \%$ & 0.91 & $25.44 \%$ & $10.29 \%$ & 0.27 \\
\hline & $-7.52 \%$ & $18.64 \%$ & -0.52 & $3.83 \%$ & 1.10 & $-8.81 \%$ & $6.42 \%$ & 0.60 \\
\hline \multirow{2}{*}{ CSA } & $24.88 \%$ & $24.01 \%$ & 0.97 & $-1.51 \%$ & $1.11^{* \star *}$ & $21.02 \%$ & $4.54 \%$ & -0.33 \\
\hline & $-10.39 \%$ & $16.51 \%$ & -0.76 & $0.12 \%$ & $1.03^{*}$ & $-12.17 \%$ & $1.62 \%$ & 0.07 \\
\hline \multirow{2}{*}{ IST } & $26.59 \%$ & $22.78 \%$ & 1.10 & $1.77 \%$ & 1.04 & $24.08 \%$ & $5.20 \%$ & 0.34 \\
\hline & $-9.11 \%$ & $16.28 \%$ & -0.69 & $1.07 \%$ & 1.00 & $-11.22 \%$ & $2.99 \%$ & 0.36 \\
\hline \multirow{2}{*}{ PREVISTA } & $24.90 \%$ & $21.60 \%$ & 1.08 & $1.44 \%$ & 0.98 & $23.85 \%$ & $6.15 \%$ & 0.23 \\
\hline & $-10.30 \%$ & $16.00 \%$ & -0.78 & $-0.14 \%$ & 1.00 & $-12.43 \%$ & $1.04 \%$ & -0.14 \\
\hline \multirow{2}{*}{ UBS } & $23.29 \%$ & $21.75 \%$ & 1.00 & $-1.10 \%$ & 1.02 & $21.30 \%$ & $1.77 \%$ & -0.62 \\
\hline & $-11.17 \%$ & $16.16 \%$ & -0.83 & $-0.97 \%$ & 1.01 & $-13.25 \%$ & $1.96 \%$ & -0.49 \\
\hline \multirow{2}{*}{ SPI } & $23.93 \%$ & $21.24 \%$ & 1.05 & $0.00 \%$ & 1.00 & $22.38 \%$ & $0.00 \%$ & - \\
\hline & $-10.12 \%$ & $15.93 \%$ & -0.77 & $0.00 \%$ & 1.00 & $-12.28 \%$ & $0.00 \%$ & \\
\hline
\end{tabular}

1. Subperiode: 1. $1996-4.1999$, respektive 5. $1996-4.1999$ für AWI.

2. Subperiode: 5. 1999 - 9. 2002.

***/* bedeutet Signifikanz auf einem Niveau von $1 \% / 10 \%$.

Alle Werte sind annualisiert.

Die Ergebnisse der beiden Subperioden fallen höchst unterschiedlich aus: Der SPI gewann in den ersten dreieinhalb Jahren fast $24 \%$ pro Jahr, während er in der zweiten Hälfte der Stichprobe $10.12 \%$ pro Jahr verlor. Auch die Volatilität liegt in der ersten Subperiode mit $21.24 \%$ deutlich höher als mit 15.93\% in der zweiten. Diese Unterschiede in den Renditezeitreihen zeigen sich bei sämtlichen Anlagestiftungen. Keine Anlagestiftung hat ein signifikant positives JENSEN's Alpha erzielt, doch zeigen immerhin Bâloise und IST in beiden Subperioden positive Alphas.[7] Die für die Alphas zusätzlich berechnete Rangkkorrelation beträgt 0.6 und ist gemäss dem SPEARMAN Rangkorrelationstest für kleine Stichproben nach KANJI (1999) S. 93 auf einem Niveau von 10\% statistisch signifikant. Dies bedeutet, dass die besten respektive schlechtesten Anlagestiftungen in der ersten Subperiode tendenziell auch in der zweiten Subperiode unter den besten respektive schlechtesten platziert waren.

Die Betas von AWI und CSA sind in der ersten Subperiode wie auch für die gesamte Zeitreihe signifikant grösser als eins. Diesen beiden Anlagestiftungen ist es auch gelungen, ihr Beta für die zweite Subperiode mit dem fallenden Aktienmarkt zu senken. Dieser Befund ist jedoch nicht mit positiven Timing-Fähigkeiten gleichzusetzen, wie das negative Gamma der CSA in Abschnitt 3.3 zeigt. 
Tabelle 10: Persistenz für ,Aktien Ausland“

\begin{tabular}{|c|c|c|c|c|c|c|c|c|}
\hline & Rendite & Volatilität & $\begin{array}{c}\text { SHARPE } \\
\text { Ratio }\end{array}$ & $\begin{array}{c}\text { JENSEN's } \\
\text { Alpha }\end{array}$ & Beta & $\begin{array}{c}\text { TREYNOR } \\
\text { Ratio } \\
\end{array}$ & $\begin{array}{c}\text { Tracking } \\
\text { Error }\end{array}$ & $\begin{array}{c}\text { Information } \\
\text { Ratio }\end{array}$ \\
\hline \multirow{2}{*}{ AWI } & $20.97 \%$ & $20.57 \%$ & 0.94 & $-4.31 \%$ & 0.98 & $19.79 \%$ & $6.60 \%$ & -0.65 \\
\hline & $-13.89 \%$ & $20.99 \%$ & -0.76 & $0.18 \%$ & 0.98 & $-16.40 \%$ & $6.18 \%$ & 0.03 \\
\hline \multirow{2}{*}{ Bâloise } & $23.78 \%$ & $18.93 \%$ & 1.17 & $4.46 \%$ & $0.73^{* * *}$ & $30.24 \%$ & $12.09 \%$ & 0.37 \\
\hline & $-16.06 \%$ & $21.71 \%$ & -0.84 & $-1.67 \%$ & 1.00 & $-18.25 \%$ & $7.24 \%$ & -0.23 \\
\hline \multirow{2}{*}{ CSA } & $23.85 \%$ & $19.06 \%$ & 1.17 & $-0.66 \%$ & $0.95^{* *}$ & $23.48 \%$ & $2.73 \%$ & -0.24 \\
\hline & $-16.03 \%$ & $21.65 \%$ & -0.84 & $-0.90 \%$ & 1.04 & $-17.45 \%$ & $3.49 \%$ & -0.26 \\
\hline \multirow{2}{*}{ IST } & $26.43 \%$ & $20.63 \%$ & 1.21 & $0.31 \%$ & 1.02 & $24.48 \%$ & $4.26 \%$ & 0.07 \\
\hline & $-15.53 \%$ & $20.95 \%$ & -0.84 & $-0.85 \%$ & 1.02 & $-17.42 \%$ & $2.42 \%$ & -0.35 \\
\hline \multirow{2}{*}{ PREVISTA } & $24.70 \%$ & $19.88 \%$ & 1.16 & $-0.18 \%$ & 0.96 & $23.99 \%$ & $5.31 \%$ & -0.03 \\
\hline & $-15.63 \%$ & $21.37 \%$ & -0.83 & $-1.00 \%$ & 1.01 & $-17.57 \%$ & $5.17 \%$ & -0.19 \\
\hline \multirow{2}{*}{ UBS } & $24.80 \%$ & $19.79 \%$ & 1.17 & $-0.70 \%$ & 0.99 & $23.47 \%$ & $2.28 \%$ & -0.30 \\
\hline & $-15.45 \%$ & $20.66 \%$ & -0.85 & $-0.93 \%$ & 1.01 & $-17.51 \%$ & $1.22 \%$ & -0.76 \\
\hline \multirow{2}{*}{ MSCI World } & $25.73 \%$ & $19.86 \%$ & 1.22 & $0.00 \%$ & 1.00 & $24.17 \%$ & $0.00 \%$ & \\
\hline & $-14.42 \%$ & $20.50 \%$ & -0.81 & $0.00 \%$ & 1.00 & $-16.58 \%$ & $0.00 \%$ & - \\
\hline \multicolumn{9}{|c|}{$\begin{array}{l}\text { 1. Subperiode: } 1.1996-4.1999 . \\
\text { 2. Subperiode: } 5.1999-9.2002 . \\
\star * \star * * \\
\text { Alle Wedeutet Signifikanz auf einem Niveau von } 1 \% / 5 \% \text {. } \\
\end{array}$} \\
\hline
\end{tabular}

Die Resultate für die „Aktien Ausland“ finden sich in Tabelle 10. Auch hier ist kein JENSEN's Alpha signifikant von null verschieden. Der SPEARMAN Rangkorrelationstest zeigt einen Wert von -0.6, was auf keine Korrelation zwischen den Rängen hinweist. Dies wird vor allem durch AWI und Bâloise verursacht, die je einmal auf dem ersten respektive auf dem letzten Platz liegen. Die Betas von Bâloise und CSA sind in der ersten Subperiode signifikant geringer als 1 - doch gerade in dieser Phase der steigenden Aktienmärkte wäre ein Beta grösser als 1 wünschenswert.

Das hier vorgenommene Verfahren zur Persistenzanalyse kann nicht zuletzt wegen der geringen Stichprobenzahl höchstens als erste Approximation betrachtet werden. Es lässt sich damit nicht abschliessend beurteilen, ob die Performance bei der vorliegenden Zeitreihe kurz- oder mittelfristig prognostiziert werden kann. Andere empirische Studien stützen sich denn auch auf deutlich längere Perioden, als sie hier vorliegen. GRINBLATT und TITMAN (1992) unterteilen die Renditezeitreihe von zehn Jahren in zwei Fünfjahres-Subperioden. CARHART (1997) stützt sich auf die Methodologie von HENDRICKS et al. (1993) und generiert Portfolios, basierend auf Einjahres-Lag Returns, für die er die Performance schätzt. Seine Zeitreihe umfasst monatliche Returns von 1963 - 1993.

\section{Schlussfolgerungen}

Für sechs Anlagestiftungen wurden bei den Sondervermögen „Aktien Schweiz“ und „Aktien Ausland" die Rendite- und Risikostruktur untersucht. Sie weisen in den Kennzahlen relativ grosse Renditedifferenzen aus, während sich die Volatilitäten und das systematische Risiko gemessen am Beta nur wenig unterscheiden. Einen vergleichsweise stark aktiven Ansatz verfolgt offensichtlich die Bâloise Anlagestiftung, wie an den hohen Tracking Errors erkennbar ist. Generell sind die Performancekennzahlen für die Sondervermögen „Aktien Schweiz“ deutlich besser als für die Sondervermögen ,Aktien Ausland“. Allerdings sind die JENSEN's Alphas in keinem Fall signifikant von null verschieden. Eine systematische Selektionsfähigkeit kann also nicht nachge- 
wiesen werden. Ebenso kann mit dem HENRIKSSON-MERTON-Modell keine systematische Timingfähigkeit nachgewiesen werden: Die Timingkoeffizienten sind in keinem Fall signifikant positiv, aber in einigen Fällen signifikant negativ.

Die Faktoranalysen zeigen grundsätzlich geringe zeitliche Verschiebungen in der Aufteilung auf Value- und Growth-Stil für die „Aktien Schweiz“, respektive Nordamerika, Europa und Asien für die „Aktien Ausland“. Im September 2001 kommt es jedoch zu grösseren Veränderungen. Die Bâloise Anlagestiftung zeigt auch hier eine eigene Strategie, was sich im Vergleich zu den übrigen Anlagestiftungen in unterschiedlichen Anteilen der einzelnen Anlagestile äussert. Gemessen an der Selektionsrendite wurde untersucht, ob die Fondsperformance positiv von den Faktorrenditen abweicht. Es gelang jedoch keiner Anlagestiftung, durch überdurchschnittliche Selektionsfähigkeiten eine systematische Outperformance zu erzielen. Allerdings konnte auch keine systematische Underperformance nachgewiesen werden.

Zusätzlich wurden die Zeitreihen in zwei gleich lange Subperioden unterteilt, um die Persistenz in der Performance der Anlagestiftungen zu untersuchen. Die erste Subperiode zeichnet sich durch stark ansteigende Aktienmärkte aus, während die zweiten Hälfte durch starke Verluste geprägt ist. Anhand der JENSEN's Alphas konnte keine Persistenz in der Performance der Anlagestiftungen nachgewiesen werden.

Zusammenfassend kann festgehalten werden, dass die hier untersuchten Anlagestiftungen keine statistisch signifikante Out- oder Underperformance erzielen, wobei mit Schweizer Aktien tendenziell bessere Ergebnisse erzielt werden als mit ausländischen Aktien. Angesichts der Kosten im Zusammenhang mit Management und Administration der Sondervermögen kann dies bereits als Erfolg gedeutet werden. Andererseits kann insbesondere bei den Sondervermögen „Aktien Ausland" die Frage gestellt werden, ob diese Resultate nicht auch mit explizit passiven - und somit kostengünstigeren - Anlagestrategien zu erzielen wären.

\section{ANHANG: VERWENDETE ABKÜRZUNGEN DER KGAST-MITGLIEDER}

AWI

Bâloise

CSA

ethos

IST

LOFI

Patria

Prevista

Sarasin

Swiss Life

UBS

Zürich
Anlagestiftung Winterthur für Personalvorsorge

Bâloise-Anlagestiftung für Personalvorsorge

Credit Suisse Anlagestiftung 2. Säule

ethos, Schweizerische Anlagestiftung für nachhaltige Entwicklung

Investmentstiftung für Personalvorsorge

Anlagestiftung Lombard Odier

Patria Anlagestiftung

Anlagestiftung für Personalvorsorgeeinrichtungen

Sarasin Anlagestiftung

Anlagestiftung Swiss Life

Anlagestiftung der UBS für Personalvorsorge

"Zürich" Anlagestiftung 


\section{FUSSNOTEN}

[1] Beispiele für Untersuchungen amerikanischer Aktienfonds sind TREYNOR (1965), SHARPE (1966), JENSEN (1968), TREYNOR und BLACK (1973), BRINSON et al. (1986), SHARPE (1988), BRINSON et al. (1991), GRINBLATT und TITMAN (1992), SHARPE (1992), HENDRICKS et al. (1993), GOETZMANN und IBBOTSON (1994) und CARHART (1997). Beispiele für Untersuchungen Schweizerischer Aktienfonds sind ZIMMERMANN und ZOGGWETTER (1992) und VON WYSS (2001).

[2] Die Ankündigung des Bundesrates am 3. Juli 2002, den Mindestzinssatz für Pensionskassen in Art. 12 BVV 2 von 4\% auf 3\% (später korrigiert auf 31\%4\%) zu senken, entfachte diese Diskussion zusätzlich.

[3] Bereinigung des Gesamtvermögens um die einer Anlagegruppe zugeflossenen Mittel, welche in Ansprüche der übrigen Anlagegruppen derselben Stiftung angelegt wurden.

[4] SHARPE schlägt ein Asset-Class-Faktormodell mit zwölf Faktoren vor (u. a. Small-, Medium-Cap sowie Value und Growth).

[5] Für einen Vergleich von Berechnungen der Gewichte mittels multipler Regression und quadratischer Programmierung vgl. MAAG (1999). Bei der multiplen Regression fallen einige Koeffizienten negativ aus, was der realisierten Asset-Allocation widerspricht. Trotzdem sind die Übereinstimmungen der mit beiden Verfahren berechneten Koeffizienten zum Teil gross. Das $\mathrm{R}^{2}$ fällt bei der multiplen Regression leicht höher aus.

[6] Aufgrund der Umparametrisierung des Modells kann grundsätzlich ein $\mathrm{R}^{2}$ berechnet werden. Im vorliegenden Fall wird die OLS Schätzung allerdings ohne Konstante durchgeführt. Deshalb können sich bei der Interpretation der $\mathrm{R}^{2}$-Werte Probleme ergeben: im Vergleich zu einer Regression mit Konstante können die $\mathrm{R}^{2}$-Werte deutlich tiefer ausfallen.

[7] Wegen der unterschiedlichen Steigungen der Regressionsgeraden (Betas) in den beiden Subperioden müssen die Alphas der Gesamtperiode nicht dem Durchschnitt der Alphas aus den Subperioden entsprechen.

\section{LITERATUR}

BRINSON, G.P., R. HOOD and G.L. BEEBOWER (1986): „Determinants of Portfolio Performance”, Financial Analysts Journal, S. 39-44.

BRINSON, G.P., B.D. SINGER and G.L. BEEBOWER (1991): „Determinants of Portfolio Performance II: An Update", Financial Analysts Journal, S. 40-48.

CARHART, M.M. (1997): „On Persistence in Mutual Fund Performance”, Journal of Finance, S. 57-82.

CHRISTOPHERSON, J.A., W.E. FERSON und D.A GLASSMAN (1998): „Conditioning Managers Alphas on Economic Information: Another Look at the Persistence of Performance“, The Review of Financial Studies, S. 111-142.

GOETZMANN, W.N. und R.G. IBBOTSON (1994): „Do Winners Repeat“, Journal of Portfolio Management, Nr. 2, S. 9-18.

GRINBLATT, M. (1987): „How to Evaluate a Portfolio Manager”, Finanzmarkt und Portfolio Management, S. 9-20. 
GRINBLATT, M. and S. TITMAN (1992): „The Persistence of Mutual Fund Performance”, Journal of Finance, S. 1977-1984.

HENDRICKS, D., J. PATEL and R. ZECKHAUSER (1993): „Hot Hands in Mutual Funds: Short-Run Persistence of Relative Performance, 1974-1988”, Journal of Finance, S. 93-130. HENRIKSSON, R. and R. MERTON (1981): „On Market Timing and Investment Performance. II. Statistical Procedures for Evaluating Forecasting Skills", Journal of Business, S. 513-533. JENSEN, M. C. (1968): „The Performance of Mutual Funds in the Period 1945-1964”, Journal of Finance, S. 389-416.

KANJI, G.K. (1999): „100 Statistical Tests“, London: SAGE.

LUCAS, L. Und M.W. RIEPE (1996): „The Role of Returns-Based Style Analysis: Understanding, Implementing, and Interpreting the Technique“, Working Paper, Chicago: Ibbotson Associates.

LUSENTI, G. (Hrsg.) (2002): 3. Umfrage über Pensionskassenanlagen 2000 - 2003: Ausgewählte Ergebnisse und Kommentare, Robeco (Schweiz) AG. http://www.vorsorge forum.ch/DE/ Statistik/Robeco/Umfrage\%202002.pdf.

MAAG, F. (1999): „Performance-Messung von Bondfonds“, Bern: Haupt.

MÜLLER, C. (2002a): „Mitteilung der KGAST“. http://www.kgast.ch.

MÜLLER, U. (2002b): „A Useful Reparametrization for Sharpe's Style Regression“, Working Paper, Schweizerisches Institut für Aussenwirtschaft und Angewandte Wirtschaftsforschung (SIAW), Universität St. Gallen.

o. V. (1982): „Bundesgesetz vom 25. Juni 1982 über die berufliche Alters-, Hinterlassenen- und Invalidenvorsorge (BVG)“', SR 831.40.

o. V. (1984): „Verordnung vom 18. April 1984 über die berufliche Alters-, Hinterlassenen- und Invalidenvorsorge (BVV 2)“, SR 831.441.1.

SHARPE, W.F. (1966): „Mutual Fund Performance”, Journal of Business, S. 119-138.

SHARPE, W.F. (1988): „Determining a Fund's Effective Asset Mix”, Investment Management Review, December, S. 59-69.

SHARPE, W.F. (1992): „Asset allocation: Management style and performance measurement”, Journal of Portfolio Management, Nr. 2, S. 7-19.

TREYNOR, J.L. (1965): „How to Rate Management of Investment Funds”, Harvard Business Review, S. 63-75.

TREYNOR, J.L. and F. BLACK (1973): „How to Use Security Analysis to Improve Portfolio Selection", Journal of Business, S. 66-86.

WATSON WYATT AG (2002a): „Performance-Vergleich per 31. Dezember 2001“. http://www.kgast.ch/de/performance.cfm?Sprache $=1$.

WATSON WYATT AG (2002b): „Performance-Vergleich per 30. Juni 2002“. http://www.kgast.ch/de/performance.cfm? Sprache=1.

WITTROCK, C. (1995): „Der Einsatz von Asset-Allocation-Modellen in der Portfolioanalyse”, Finanzmarkt und Portfolio Management, S. 361-383.

VON WYSS, R. (2001): „Performance Measurement of Equity Funds - Do the SPPS Enhance Transparency?“, Financial Markets and Portfolio Management, S. 173-186.

ZIMMERMANN, H., M. RUDOLF, S. JAEGER und C. ZOGG-WETTER (1996): „Moderne Performance-Messung. Ein Handbuch für die Praxis“, Bern: Haupt.

ZIMMERMANN, H. und C. ZOGG-WETTER (1992): „Performance-Messung schweizerischer Aktienfonds: Markt-Timing und Selektivität“, Schweizerische Zeitschrift für Volkswirtschaft und Statistik, S. 133-160. 